
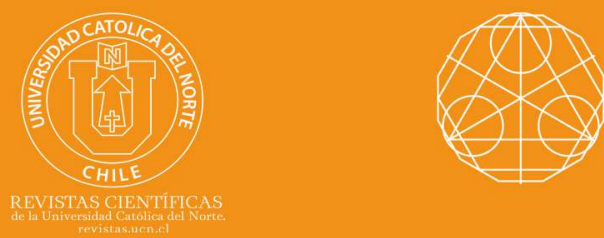

\title{
Lacunary sequences of complex uncertain variables defined by Orlicz functions
}

Pranab Jyoti Dowari ${ }^{1}$

Binod Chandra Tripathy² ${ }^{2}$ orcid.org/0000-0002-0738-652X

Tripura University, Dept. of Mathematics, Agartala, TR, India.

$1 \bowtie$ pranabdowari@gmail.com ; ${ }^{2}$ tripathybc@rediffmail.com

\section{Abstract:}

Using the concept of Orlicz function and uncertainty theory, some new class of lacunary convergent sequences defined by Orlicz functions have been introduced with the lacunary convergence concepts in this paper. Some topological properties of the defined sequence spaces along with the inclusion relations have been investigated.

Keywords: Almost convergent sequences; Complex uncertain variable; Orlicz function; Lacunary sequence.

MSC (2020): 40A05; 40A25; 60B10; 60B12; 60F17.

\section{Cite this article as (IEEE citation style):}

P. J. Dowari and B. C. Tripathy, "Lacunary sequences of complex uncertain variables defined by Orlicz functions", Proyecciones (Antofagasta, On line), vol. 40, no. 2, pp. 355-370, 2021, doi: 10.22199/issn.0717-6279-2021-02-0021

Article copyright: (C) 2021 Pranab Jyoti Dowari and Binod Chandra Tripathy. This is an open access article distributed under the terms of the Creative Commons License, which permits unrestricted use and distribution provided the original author and source are credited. 


\section{Introduction and preliminaries}

The notion of uncertainty theory was introduced by Liu [9] in the year 2007 which is based on an uncertain measure which satisfies normality, duality, subadditivity and product axioms. The concepts of uncertainty theory have been investigated from different aspects with applications. The notion of complex uncertain sequence spaces of uncertain variables was studied by Tripathy and Nath [6]. Further uncertain programming, uncertain risk analysis, uncertain logic etc. have been studied. Liu [9] first introduced the convergence concepts of sequences of uncertain variables. Later on Chen et al. [16], You [10], Dowari and Tripathy [13], Liu [7] and many others further studied the convergence of sequences of uncertain variables. The notion of lacunary sequences was initiated by Freedman et al. [1]. Their studies of the $\left|\sigma_{1}\right|$ of strongly Cesàro summable sequences with general lacunary $\theta$ resulted in introducing a larger class of sequences $N_{\theta}$ called lacunary sequences. Later their work have been further extended by many researchers. The prime interest of this current study is to extend the classes with the uncertain sequences in the uncertainty space and the lacunary convergence concepts of complex uncertain sequences with respect to an Orlicz function.

An Orlicz function is a function $M:[0, \infty) \rightarrow[0, \infty)$ which is continuous, non-decreasing and convex with $M(0)=0, M(x)>0$ for $x>0$, and $M(x) \rightarrow \infty$ as $x \rightarrow \infty$.

An Orlicz function can be represented in the integral form as follows-

$$
M(x)=\int_{0}^{x} p(t) d t
$$

where $\mathrm{p}$, known as the kernel of $M$ is right differentiable for $t \geq 0, p(0)=$ $0, p(t)>0$ for $t>0, p$ is non-decreasing and $p(t) \rightarrow \infty$ as $t \rightarrow \infty$. If the convexity of an Orlicz function $M$ is replaced by its subadditivity, i.e. $M(x+y)=M(x)+M(y)$, then this function is called a modulus function. Lindenstrauss and Tzafriri[14] used the idea of the Orlicz function to construct the sequence space

$$
\ell_{M}=\left\{\left(x_{k}\right) \in w: \sum_{k=1}^{\infty} M\left(\frac{\left|x_{k}\right|}{\rho}\right)<\infty, \text { for some } \rho>0\right\},
$$

where $w$ denotes the class of all sequences.

The space $\ell_{M}$ with the norm

$$
\|x\|=\inf \left\{\rho>0: \sum_{k=1}^{\infty} M\left(\frac{\left|x_{k}\right|}{\rho}\right) \leq 1\right\},
$$


becomes a Banach space which is called an Orlicz sequence space. The space $\ell_{M}$ is closely related to the space $\ell_{p}$ which is an Orlicz sequence space with $M(x)=|x|^{p}$ for $1 \leq p<\infty$.

In the subsequent time, different Orlicz sequence spaces were introduced by Parashar and Choudhury [15], Tripathy and Mahanta [2, 3], Tripathy and Esi [4] and many others.

In this we define the sequence spaces of uncertain variables defined by Orlicz functions and establish an inclusion relation between these spaces.

We now give here a brief introduction of the uncertainty theory. Let $\mathcal{L}$ be a $\sigma$-algebra on a nonempty set $\Gamma$. A set function $\mathcal{M}$ is called an uncertain measure if it satisfies the following axioms:

Axiom 1 (Normality Axiom). $\mathcal{M}\{\Gamma\}=1$;

Axiom 2 (Duality Axiom). $\mathcal{M}\{\Lambda\}+\mathcal{M}\left\{\Lambda^{c}\right\}=1$ for any $\Lambda \in \mathcal{L}$;

Axiom 3 (Subadditivity Axiom). For every countable sequence of $\left\{\lambda_{j}\right\} \in$ $\mathcal{L}$, we have

$$
\mathcal{M}\left\{\bigcup_{j=1}^{\infty} \lambda_{j}\right\} \leq \sum_{j=1}^{\infty} \mathcal{M}\left\{\lambda_{j}\right\} .
$$

The triplet $(\Gamma, \mathcal{L}, \mathcal{M})$ is called an uncertainty space, and each element $\Lambda$ in $\mathcal{L}$ is called an event. In order to obtain an uncertain measure of compound event, a product uncertain measure is defined by Liu [9] as follows:

Axiom 4 (Product Axiom). Let $\left(\Gamma_{k}, \mathcal{L}_{k}, \mathcal{M}_{k}\right)$ be uncertainty space for $k=1,2,3, \ldots$ The product uncertain measure $\mathcal{M}$ is an measure satisfying

$$
\mathcal{M}\left\{\prod_{k=1}^{\infty} \Lambda_{k}\right\}=\bigwedge_{k=1}^{\infty} \mathcal{M}_{k}\left\{\Lambda_{k}\right\}
$$

where $\Lambda_{k}$ are arbitrarily chosen events from $\mathcal{L}_{k}$ for $k=1,2, \ldots$, respectively. 
A complex uncertain variable is a measurable function $\xi$ from an uncertainty space $(\Gamma, \mathcal{L}, \mathcal{M})$ to the set of complex numbers, i.e., for any Borel set $\mathcal{B}$ of complex numbers, the set

$$
\{\xi \in \mathcal{B}\}=\{\gamma \in \Gamma: \xi(\gamma) \in \mathcal{B}\}
$$

is an event. When the range is the set of real numbers, we call it as an uncertain variable, introduced and investigated by Liu[9]. As a complex function on uncertainty space, complex uncertain variable is mainly used to model a complex uncertain quantity.

The expected value operator of an uncertain variable was defined by $\mathrm{Liu}[9]$ as

$$
E[\xi]=\int_{0}^{+\infty} \mathcal{M}\{\xi \geq r\} d r-\int_{-\infty}^{0} \mathcal{M}\{\xi \leq r\} d r
$$

provided that at least one of the two integrals is finite.

The complex uncertainty distribution $\Phi(x)$ of a complex uncertain variable $\xi$ is a function from $\mathbf{C}$ to $[0,1]$ defined by

$$
\Phi(c)=\mathcal{M}\{\operatorname{Re}(\xi) \leq \operatorname{Re}(c), \operatorname{Im}(\xi) \leq \operatorname{Im}(c)\}
$$

for any complex number $c$.

An uncertain variable is said to be positive, when it maps from $\mathbf{R}_{+} \bigcup\{0\}$ (non-negative real numbers) to $[0,1]$. Considering the important role of sequence convergence in mathematics, some concepts of convergence for complex uncertain sequences were introduced in Chen et al. [16]. Complex uncertain sequences are sequence of complex uncertain variables indexed by integers.

The complex uncertain sequence $\left\{\xi_{n}\right\}$ is said to be convergent almost surely(a.s.) to $L$ if there exists an event $\Lambda$ with $\mathcal{M}\{\Lambda\}=1$ such that

$$
\lim _{n \rightarrow \infty}\left\|\xi_{n}(\gamma)-L(\gamma)\right\|=0
$$

for every $\gamma \in \Lambda$. In that case we write $\xi_{n} \rightarrow L$, a.s..

The complex uncertain sequence $\left\{\xi_{n}\right\}$ is said to be convergent in measure to $L$ if for a given $\varepsilon>0$,

$$
\lim _{n \rightarrow \infty} \mathcal{M}\left\{\left\|\xi_{n}(\gamma)-L(\gamma)\right\| \geq \varepsilon\right\}=0 .
$$


The complex uncertain sequence $\left\{\xi_{n}\right\}$ is said to be convergent in mean to $L$ if

$$
\lim _{n \rightarrow \infty} E\left[\left\|\xi_{n}(\gamma)-L(\gamma)\right\|\right]=0 .
$$

Let $\Phi_{1}, \Phi_{2}, \Phi_{3}, \ldots$ be the complex uncertainty distributions of complex uncertain variables $\xi_{1}, \xi_{2}, \xi_{3} \ldots$, respectively. We say the complex uncertain sequence $\left\{\xi_{n}\right\}$ converges in distribution to $L$ if

$$
\lim _{n \rightarrow \infty} \Phi_{n}(c)=\Phi(c)
$$

for all $c \in \mathbf{C}$, at which $\Phi(c)$ is continuous.

The complex uncertain sequence $\left\{\xi_{n}\right\}$ is said to be convergent uniformly almost surely(u.a.s.) to $L$ if there exists a sequence of events $\left\{E_{k}^{\prime}\right\}$, $\mathcal{M}\left\{E_{k}^{\prime}\right\} \rightarrow 0$ such that $\left\{\xi_{n}\right\}$ converges uniformly to $L$ in $\Gamma-E_{k}^{\prime}$, for any fixed $k \in \mathbf{N}$.

\section{Definition and Notations}

In this section we define some new classes of sequences of uncertain variables using an Orlicz functions. By a lacunary sequence $\theta=\left(k_{r}\right)$; where $k_{0}=0$ we shall mean an increasing sequence of non-negative integers with $k_{r}-k_{r-1} \rightarrow \infty$, as $r \rightarrow \infty$. The intervals determined by $\theta$ will be denoted by $I_{r}=\left(k_{r-1}, k_{r}\right]$ and $q_{r}=\frac{k_{r}}{k_{r-1}}, h_{r}=k_{r}-k_{r-1}$ for $r=1,2,3, \ldots$

Sums of the form $\sum_{i=k_{r-1}+1}^{k_{r}}\left|x_{i}\right|=\sum_{i \in I_{r}}\left|x_{i}\right|$, will often be denoted by $\sum_{I_{r}}\left|x_{i}\right|$ for convenience.

Here we define the space $\left|\sigma_{1}\right|^{U}$ of strongly Cesàro Summable uncertain sequence as

$\left|\sigma_{1}\right|^{U}=\left\{\xi=\left(\xi_{k}(\gamma)\right)\right.$ :there exists $L(\gamma)$ such that $\frac{1}{n} \sum_{i=1}^{n}\left\|\xi_{i}(\gamma)-L(\gamma)\right\| \rightarrow 0$, as $n \rightarrow \infty\}$.

Let $\theta=\left(k_{r}\right)$ be a lacunary sequence and $\left(\xi_{k}\right)$ be a sequence of uncertain variables in the space $(\Gamma, \mathcal{L}, \mathcal{M}), M$ be an Orlicz function. We define the following class of sequences,

$$
\left[\mathrm{N}_{\theta}^{U}, M\right]_{0}=\left\{\xi=\left(\xi_{k}\right): \lim _{r \rightarrow \infty} \frac{1}{h_{r}} \sum_{k \in I_{r}} M\left(\frac{\left\|\xi_{k}(\gamma)\right\|}{\rho}\right)=0 \text {, for some } \rho>0\right\} .
$$




$$
\begin{gathered}
{\left[N_{\theta}^{U}, M\right]_{1}=\left\{\xi=\left(\xi_{k}\right): \lim _{r \rightarrow \infty} \frac{1}{h_{r}} \sum_{k \in I_{r}} M\left(\frac{\left\|\xi_{k}(\gamma)-L(\gamma)\right\|}{\rho}\right)=0,\right. \text { for some }} \\
\\
\rho>0 \text { and } L(\gamma) \in(\Gamma, \mathcal{L}, \mathcal{M})\} . \\
{\left[\mathrm{N}_{\theta}^{U}, M\right]_{\infty}=\left\{\xi=\left(\xi_{k}\right): \sup _{r} \frac{1}{h_{r}} \sum_{k \in I_{r}} M\left(\frac{\left\|\xi_{k}(\gamma)\right\|}{\rho}\right)<\infty, \text { for some } \quad \rho>0\right\} .}
\end{gathered}
$$

Let $\lambda$ be a sequence space. Then $\lambda$ is called

(I) A sequence space $\lambda$ is said to be symmetric if $\left(x_{k}\right) \in \lambda \Rightarrow\left(x_{\pi(k)}\right) \in \lambda$, $\pi$ is a permutation of $\mathbf{N}$.

(II) Monotone: A sequence space $\lambda$ is said to be monotone if $E$ contains the canonical pre-images of all its step spaces.

(III) Normal or Solid: A sequence space $\lambda$ is said to be solid if $y \in \lambda$ whenever $\left\|y_{k}\right\| \leq\left\|x_{k}\right\|, \quad k \in \mathbf{N}$ for all $x \in \lambda$.

Lemma 2.1. A sequence space $\lambda$ is solid implies $\lambda$ is monotone.

\section{The main results}

In this section we examine the basic properties of the spaces $\left[N_{\theta}^{U}, M\right]_{0},\left[N_{\theta}^{U}, M\right]_{1},\left[N_{\theta}^{U}, M\right]_{\infty}$ and obtain the inclusion relation for these spaces.

Theorem 3.1. The classes of complex uncertain sequences $\left[N_{\theta}^{U}, M\right]_{0},\left[N_{\theta}^{U}, M\right]_{1}$ and $\left[N_{\theta}^{U}, M\right]_{\infty}$ are linear spaces.

Proof. We prove the result for the class of complex uncertain sequence $\left[N_{\theta}^{U}, M\right]_{\infty}$. The other cases can be proved in the similar manner. Let $\left(\xi_{k}\right),\left(\eta_{k}\right) \in\left[N_{\theta}^{U}, M\right]_{\infty}$ and $\alpha, \beta \in \mathbf{C}$. Then there exists positive numbers $\rho_{1}, \rho_{2}>0$ such that

$$
\sup _{r} \frac{1}{h_{r}} \sum_{k \in I_{r}} M\left(\frac{\left\|\xi_{k}(\gamma)\right\|}{\rho_{1}}\right)<\infty
$$

and

$$
\sup _{r} \frac{1}{h_{r}} \sum_{k \in I_{r}} M\left(\frac{\left\|\eta_{k}(\gamma)\right\|}{\rho_{2}}\right)<\infty .
$$

Define $\rho_{3}=\max \left(2|\alpha| \rho_{1}, 2|\beta| \rho_{2}\right)$.

Since $M$ is non-decreasing and convex, we have 


$$
\begin{aligned}
& \sum_{k \in I_{r}} M\left[\left(\frac{\left\|\alpha \xi_{k}(\gamma)+\beta \eta_{k}(\gamma)\right\|}{\rho_{3}}\right)\right] \\
& \leq \sum_{k \in I_{r}} M\left[\frac{\left\|\alpha \xi_{k}(\gamma)\right\|}{\rho_{3}}+\frac{\left\|\beta \eta_{k}(\gamma)\right\|}{\rho_{3}}\right] \\
& \leq \sum_{k \in I_{r}} \frac{1}{2}\left[M\left(\frac{\left\|\xi_{k}(\gamma)\right\|}{\rho_{1}}\right)+M\left(\frac{\left\|\eta_{k}(\gamma)\right\|}{\rho_{2}}\right)\right] \\
& <\sum_{k \in I_{r}} M\left(\frac{\left\|\xi_{k}(\gamma)\right\|}{\rho_{1}}\right)+\sum_{k \in I_{r}} M\left(\frac{\left\|\eta_{k}(\gamma)\right\|}{\rho_{2}}\right) \\
& \Rightarrow \sup _{r} \frac{1}{h_{r}} \sum_{k \in I_{r}} M\left[\frac{\left\|\alpha \xi_{k}(\gamma)+\beta \eta_{k}(\gamma)\right\|}{\rho_{3}}\right] \\
& \leq \sup _{r} \frac{1}{h_{r}} \sum_{k \in I_{r}} M\left(\frac{\left\|\xi_{k}(\gamma)\right\|}{\rho_{1}}\right)+\sup _{r} \frac{1}{h_{r}} \sum_{k \in I_{r}} M\left(\frac{\left\|\eta_{k}(\gamma)\right\|}{\rho_{2}}\right) \\
& <\infty \quad \Rightarrow\left(\alpha \xi_{k}(\gamma)+\beta \eta_{k}(\gamma)\right) \in\left[N_{\theta}^{U}, M\right]_{\infty} .
\end{aligned}
$$

Hence $\left[N_{\theta}^{U}, M\right]_{\infty}$ is a linear space.

Theorem 3.2. For any Orlicz function $M,\left[N_{\theta}^{U}, M\right]_{\infty}$ is a normed linear space whose norm is defined by

$$
g(\xi)=\inf \left\{\rho>0: \sup _{r} h_{r}^{-1} \sum_{k \in I_{r}} M\left(\frac{\left\|\xi_{k}\right\|}{\rho}\right) \leq 1, r=1,2,3, \ldots\right\} .
$$

The infimum is taken over all $\rho>0$.

Proof. Clearly, $g\left(\left(\xi_{k}\right)\right) \geq 0$, for all $\left(\xi_{k}\right) \in\left[N_{\theta}, M\right]_{\infty}$ and $g(\bar{\theta})=0$. Let $\rho_{1}>0$ and $\rho_{2}>0$ be such that

$$
\sup _{r} \frac{1}{h_{r}} \sum_{k \in I_{r}} M\left(\frac{\left\|\xi_{k}(\gamma)\right\|}{\rho_{1}}\right) \leq 1
$$

and

$$
\sup _{r} \frac{1}{h_{r}} \sum_{k \in I_{r}} M\left(\frac{\left\|\eta_{k}(\gamma)\right\|}{\rho_{2}}\right) \leq 1
$$

Let $\rho=\rho_{1}+\rho_{2}$. Then we have,

$$
\begin{aligned}
\sup _{r} \frac{1}{h_{r}} \sum_{k \in I_{r}} M\left(\frac{\left\|\xi_{k}(\gamma)+\eta_{k}(\gamma)\right\|}{\rho}\right) \leq & \sup _{r} \frac{1}{h_{r}} \sum_{k \in I_{r}} M\left(\frac{\left\|\xi_{k}(\gamma)+\eta_{k}(\gamma)\right\|}{\rho_{1}+\rho_{2}}\right) \\
\leq & \sup _{r} \frac{1}{h_{r}} \sum_{k \in I_{r}} \frac{\rho_{1}}{\rho_{1}+\rho_{2}} M\left(\frac{\left\|\xi_{k}(\gamma)\right\|}{\rho_{1}}\right) \\
& +\frac{\rho_{1}}{\rho_{1}+\rho_{2}} M\left(\frac{\left\|\eta_{k}(\gamma)\right\|}{\rho_{2}}\right) \\
\leq & \frac{\rho_{1}}{\rho_{1}+\rho_{2}} \sup _{r} \frac{1}{h_{r}} \sum_{k \in I_{r}} M\left(\frac{\left\|\xi_{k}(\gamma)\right\|}{\rho_{1}}\right) \\
& +\frac{\rho_{2}}{\rho_{1}+\rho_{2}} \sup _{r} \frac{1}{h_{r}} \sum_{k \in I_{r}} M\left(\frac{\left\|\xi_{k}(\gamma)\right\|}{\rho_{2}}\right) \\
\leq & 1 .
\end{aligned}
$$


Since the $\rho$ 's are non-negative, so we have,

$$
\begin{aligned}
g(x+y) & =\inf \left\{\rho>0: \sup _{r} \frac{1}{h_{r}} \sum_{r} M\left(\frac{\left\|\xi_{k}(\gamma)+\eta_{k}(\gamma)\right\|}{\rho}\right) \leq 1\right\} \\
= & \inf \left\{\rho_{1}>0: \sup _{r} \frac{1}{h_{r}} \sum_{r} M\left(\frac{\left\|\xi_{k}(\gamma)\right\|}{\rho_{1}}\right) \leq 1\right\} \\
& +\inf \left\{\rho_{2}>0: \sup _{r} \frac{1}{h_{r}} \sum_{r} M\left(\frac{\left\|\xi_{k}(\gamma)\right\|}{\rho_{2}}\right) \leq 1\right\} \\
\Rightarrow g(x+y)= & g(x)+g(y) .
\end{aligned}
$$

Now, for $\lambda \in \mathbf{C}$, assume $\lambda \neq 0$, then

$$
\begin{aligned}
& g\left(\lambda \xi_{k}(\gamma)\right)=\inf \left\{\rho>0: \sup _{r} \frac{1}{h_{r}} \sum_{r} M\left(\frac{\left\|\lambda \xi_{k}(\gamma)\right\|}{\rho}\right) \leq 1\right\} \\
& =\inf \left\{(|\lambda| r)>0: \sup _{r} \frac{1}{h_{r}} \sum_{r} M\left(\frac{\left\|\lambda \xi_{k}(\gamma)\right\|}{r}\right) \leq 1\right\} ; r=\frac{\rho}{|\lambda|} \\
& =\inf \left\{r>0: \sup _{r} \frac{1}{h_{r}} \sum_{r} M\left(\frac{\left\|\xi_{k}(\gamma)\right\|}{r}\right) \leq 1\right\} \\
& =|\lambda| g(\xi(\gamma)) \text {. }
\end{aligned}
$$

This completes the proof.

Theorem 3.3. The spaces $\left[N_{\theta}^{U}, M\right]_{0},\left[N_{\theta}^{U}, M\right]_{1}$ and $\left[N_{\theta}^{U}, M\right]_{\infty}$ are solid.

Proof. $\quad$ Let $\left(\xi_{k}\right) \in\left[N_{\theta}^{U}, M\right]_{0}$.

Then there exists $\rho>0$ such that $\lim _{r \rightarrow \infty} \frac{1}{h_{r}} \sum_{k \in I_{r}} M\left(\frac{\left\|\xi_{k}(\gamma)\right\|}{\rho}\right)=0$.

Let $\left(\alpha_{k}\right)$ be a sequence o scalars such that $\left|\alpha_{k}\right| \leq 1$.

Then for each $r$, we have

$$
\begin{array}{ll}
\frac{1}{h_{r}} \sum_{k \in I_{r}} M\left(\frac{\alpha_{k} \xi_{k}(\gamma)}{\rho}\right) & \leq \frac{1}{h_{r}} \sum_{k \in I_{r}} M\left(\frac{\xi_{k}(\gamma)}{\rho}\right) \\
\Rightarrow \lim _{r \rightarrow \infty} \sum_{k \in I_{r}} M\left(\frac{\alpha_{k} \xi_{k}(\gamma)}{\rho}\right) & =0 \\
\Rightarrow\left(\alpha_{k} \xi_{k}(\gamma)\right) \in\left[N_{\theta}^{U}, M\right]_{0} . &
\end{array}
$$

Theorem 3.4. The spaces $\left[N_{\theta}^{U}, M\right]_{0},\left[N_{\theta}^{U}, M\right]_{1}$ and $\left[N_{\theta}^{U}, M\right]_{\infty}$ are monotone.

Proof. It follows directly from the lemma 2.1.

Remark 3.1. The spaces $\left[N_{\theta}^{U}, M\right]_{0},\left[N_{\theta}^{U}, M\right]_{1}$ and $\left[N_{\theta}^{U}, M\right]_{\infty}$ are not symmetric in general.

This can be illustrated with the following example.

Example 3.1. We shall show this for $\left[N_{\theta}^{U}, M\right]_{0}$, similar examples can be constructed for the other two spaces. Let $M(x)=x$ and consider the 
lacunary sequence $\theta=\left(2^{r}\right)$. Take the uncertainty space $(\Gamma, \mathcal{L}, \mathcal{M})$ to be $\left\{\gamma_{1}, \gamma_{2}, \gamma_{3}, \ldots\right\}$ with the power set and $\Lambda \in \mathcal{L}$ such that

$$
\mathcal{M}\{\Lambda\}= \begin{cases}\sup _{\gamma_{i} \in \Lambda} \frac{i}{2 i+1}, & \text { if } \sup _{\gamma_{i} \in \Lambda} \frac{i}{2 i+1}<0.5 \\ 1-\sup _{\gamma_{i} \in \Lambda^{c}} \frac{i}{2 i+1}, & \text { if } \sup _{\gamma_{i} \in \Lambda^{c}} \frac{i}{2 i+1}<0.5 \\ 0.5, & \text { otherwise. }\end{cases}
$$

Define uncertain variables by

$$
\xi_{i}\left(\gamma_{j}\right)= \begin{cases}i, & \text { if } j=i \\ 0, & \text { otherwise }\end{cases}
$$

for $i=1,2, \ldots$ and $\xi \equiv 0$.

Then the sequence $\xi_{i}$ for all $i \in \mathbf{N}$ is in $\left[N_{\theta}^{U}, M\right]_{0}$.

Consider the sequence $\left\{\eta_{i}\right\}$ which is a rearrangement of the sequence $\left\{\xi_{i}\right\}$ defined by

$\eta_{i}(\gamma)=\left\{\xi_{1}, \xi_{5}, \xi_{9}, \xi_{2}, \xi_{10}, \ldots\right\} \notin\left[N_{\theta}^{U}, M\right]_{0}$. Thus $\left[N_{\theta}^{U}, M\right]_{0}$ are not symmetric in general.

\section{Lacunary Convergence Concepts of Complex Uncertain Sequences with respect to Orlicz function}

In this section we define the lacunary convergence concepts of uncertain sequences with respect to Orlicz function and derive the relations between them.

Definition 4.1. The complex uncertain sequence $\left\{\xi_{k}\right\}$ is said to be lacunary strongly convergent almost surely to $L$ with respect to an Orlicz function $M$ if for every $\varepsilon>0$ there exists an event $\Lambda$ with $\mathcal{M}\{\Lambda\}=1$ such that

$$
\lim _{r \rightarrow \infty} \frac{1}{h_{r}} \sum_{k \in I_{r}} M\left\|\xi_{k}(\gamma)-L(\gamma)\right\|=0,
$$

for every $\gamma \in \Lambda$.

Definition 4.2. The complex uncertain sequence $\left\{\xi_{k}\right\}$ is said to be lacunary strongly convergent in measure to $L$ with respect to an Orlicz function $M$ if

$$
\lim _{r \rightarrow \infty} \mathcal{M}\left[\left\{\gamma \in \Gamma: \frac{1}{h_{r}} \sum_{k \in I_{r}} M\left\|\xi_{k}(\gamma)-L(\gamma)\right\|>\varepsilon\right\}\right]=0,
$$

for every $\varepsilon>0$. 
Definition 4.3. The complex uncertain sequence $\left\{\xi_{k}\right\}$ is said to be lacunary strongly convergent in mean to $L$ with respect to an Orlicz function $M$ if

$$
\lim _{r \rightarrow \infty} E\left[\frac{1}{h_{r}} \sum_{k \in I_{r}} M\left\|\xi_{k}(\gamma)-L(\gamma)\right\|\right]=0
$$

for every $\varepsilon>0$.

Definition 4.4. Let $\Phi_{1}, \Phi_{2}, \Phi_{3}, \ldots$ be the complex uncertainty distributions of complex uncertain variables $\xi_{1}, \xi_{2}, \xi_{3}, \ldots$, respectively. We say the complex uncertain sequence $\left\{\xi_{k}\right\}$ lacunary strong convergent in distribution to $L$ with respect to an Orlicz function $M$ if for every $\varepsilon>0$,

$$
\lim _{r \rightarrow \infty} \frac{1}{h_{r}} \sum_{k \in I_{r}} M\left\|\Phi_{k}(c)-\Phi(c)\right\|=0
$$

for all complex $c$ at which $\Phi(c)$ is continuous.

Definition 4.5. The complex uncertain sequence $\left\{\xi_{k}\right\}$ is said to be lacunary convergent uniformly almost surely to $L$ with respect to an Orlicz function $M$ if there exists an sequence of events $\left\{E_{i}\right\}, \mathcal{M}\left\{E_{i}\right\} \rightarrow 0$ such that $\left\{\xi_{k}\right\}$ converges uniformly to $L$ in $\Gamma-E_{i}$, for any fixed $i \in \mathbf{N}$.

The relations among the convergence concepts of complex uncertain sequences are discussed below.

Theorem 4.1. If the complex uncertain sequence $\left\{\xi_{k}\right\}$ lacunary strongly convergent in mean to $L$ with respect to an Orlicz function $M$, then $\left\{\xi_{k}\right\}$ lacunary strongly converges in measure to $L$.

Proof. It follows from the Markov's inequality that for any given $\varepsilon>0$, we have

$$
\begin{gathered}
\lim _{r \rightarrow \infty} \mathcal{M}\left[\left\{\gamma \in \Gamma: \frac{1}{h_{r}} \sum_{k \in I_{r}} M\left\|\xi_{k}(\gamma)-L(\gamma)\right\|>\varepsilon\right\}\right] \\
\leq \lim _{r \rightarrow \infty} \frac{E\left[\frac{1}{h_{r}} \sum_{k \in I_{r}} M\left\|\xi_{k}(\gamma)-L(\gamma)\right\|\right]}{\varepsilon} \rightarrow 0
\end{gathered}
$$

as $r \rightarrow \infty$. Thus $\left\{\xi_{k}\right\}$ lacunary strongly converges in measure to $L$ with respect to an Orlicz function $M$ and the theorem is thus proved. 
But the converse of the above theorem is not true. i.e. lacunary strong convergence in measure with respect to an Orlicz function $M$ does not imply lacunary strong convergence in mean with respect to an Orlicz function $M$. This can be illustrated with the example below.

Example 4.1. Consider the uncertainty space $(\Gamma, \mathcal{L}, \mathcal{M})$ to be $\left\{\gamma_{1}, \gamma_{2}, \ldots\right\}$ with power set and

$$
\mathcal{M}\{\Lambda\}= \begin{cases}\sup _{\gamma_{i} \in \Lambda} \frac{1}{i}, & \text { if } \sup _{\gamma_{i} \in \Lambda} \frac{1}{i}<0.5 \\ 1-\sup _{\gamma_{i} \in \Lambda^{c}} \frac{1}{i}, & \text { if } \sup _{\gamma_{i} \in \Lambda^{c}} \frac{1}{i}<0.5 \\ 0.5, & \text { otherwise, }\end{cases}
$$

and the complex uncertain variables be defined by

$$
\xi_{i}\left(\gamma_{j}\right)= \begin{cases}i, & \text { if } j=i \\ 0, & \text { otherwise }\end{cases}
$$

for $i \in I_{r}$ and $L \equiv 0$. For $\varepsilon>0$, we have

$$
\begin{aligned}
& \lim _{r \rightarrow \infty} \mathcal{M}\left(\left\{\gamma \in \Gamma: \frac{1}{h_{r}} \sum_{k \in I_{r}} M\left\|\xi_{k}(\gamma)-L(\gamma)\right\|>\varepsilon\right\}\right) \\
& =\lim _{r \rightarrow \infty} \mathcal{M}\left(\left\{\gamma \in \Gamma: \frac{1}{h_{r}} \sum_{k \in I_{r}} M\left\|\xi_{k}(\gamma)\right\|>\varepsilon\right\}\right) \\
& =\lim _{r \rightarrow \infty} \mathcal{M}\left(\left\{\gamma_{i}\right\}\right) \\
& =\lim _{r \rightarrow \infty} \frac{1}{i} \rightarrow 0 \quad\left(\text { as } \quad i \in I_{r}\right)
\end{aligned}
$$

The sequence $\left\{\xi_{i}\right\}$ lacunary strongly converges in measure to $L$.

However for each $i \in I_{r}$, we have the uncertainty distribution of uncertain variable $\left\|\xi_{i}-L\right\|=\left\|\xi_{i}\right\|$ is

$$
\begin{gathered}
\Phi_{i}(x)= \begin{cases}0, & \text { if } x<0 ; \\
1-\frac{1}{i}, & \text { if } 0 \leq x<i ; \\
1, & \text { otherwise. }\end{cases} \\
E\left[\frac{1}{h_{r}} \sum_{k \in I_{r}} M\left\|\xi_{k}(\gamma)-L(\gamma)\right\|\right]=\int_{0}^{+\infty} \mathcal{M}\{\xi \geq x\} d x-\int_{-\infty}^{0} \mathcal{M}\{\xi \leq x\} d x \\
=\int_{0}^{i} 1-\left(1-\frac{1}{i}\right) d x \\
=1 .
\end{gathered}
$$

That is, the $\left\{\xi_{i}(\gamma)\right\}$ does not converge in mean to $L(\gamma)$ with respect to an Orlicz function $M$.

Lemma 4.1. Assume complex uncertain sequence $\left\{\xi_{n}\right\}$ with real part $\left\{\zeta_{n}\right\}$ and imaginary part $\left\{\eta_{n}\right\}$, respectively, for $n=1,2, \ldots$ If uncertain sequences $\left\{\zeta_{n}\right\}$ and $\left\{\eta_{n}\right\}$ converge in measure to $L_{1}$ and $L_{2}$ respectively, then complex uncertain sequence $\left\{\xi_{n}\right\}$ converge in measure to $L=L_{1}+i L_{2}$. 
Theorem 4.2. Assume complex uncertain sequence $\left\{\xi_{k}\right\}$ with real part $\left\{\zeta_{k}\right\}$ and imaginary $\left\{\eta_{k}\right\}$, respectively, for $k \in I_{r}$. If uncertain sequences $\left\{\zeta_{k}\right\}$ and $\left\{\eta_{k}\right\}$ lacunary strongly convergent in measure to $L_{1}$ and $L_{2}$ respectively with respect to Orlicz function $M$, then complex uncertain sequence $\left\{\xi_{k}\right\}$ lacunary strongly uniformly convergent in distribution to $L=L_{1}+i L_{2}$.

Proof. Let $c=a+i b$ be a point at which the complex uncertainty distribution $\Phi$ is continuous. For any $\alpha>a, \beta>b$, we have,

$$
\begin{aligned}
& \left\{\zeta_{k} \leq a, \eta_{k} \leq b\right\}=\left\{\zeta_{k} \leq a, \eta_{k} \leq b, \mathrm{Ł}_{1} \leq \alpha, \mathrm{Ł}_{2} \leq \beta\right\} \\
& \cup\left\{\zeta_{k} \leq a, \eta_{k} \leq b, \mathrm{Ł}_{1}>\alpha, \mathrm{七}_{2}>\beta\right\} \\
& \cup\left\{\zeta_{k} \leq a, \eta_{k} \leq b, \mathrm{E}_{1} \leq \alpha, \mathrm{七}_{2}>\beta\right\} \cup\left\{\zeta_{k} \leq a, \eta_{k} \leq b, \mathrm{七}_{1}>\alpha, \mathrm{七}_{2} \leq \beta\right\} \\
& \subset\left\{\mathrm{E}_{1} \leq \alpha, \mathrm{E}_{2} \leq \beta\right\} \cup\left\{M\left\|\zeta_{k}(\gamma)-\mathrm{E}_{1}(\gamma)\right\| \geq \alpha-a\right\} \cup\left\{M\left\|\eta_{k}(\gamma)-\mathrm{E}_{2}(\gamma)\right\| \geq \beta-b\right\} .
\end{aligned}
$$

It follows from the subadditivity axiom that

$$
\begin{aligned}
\Phi_{k}(c) & =\Phi_{k}(a+i b) \\
& \leq \Phi(\alpha+i \beta)+\mathcal{M}\left\{\gamma \in \Gamma: M\left\|\zeta_{k}(\gamma)-L_{1}(\gamma)\right\| \geq \alpha-a\right\} \\
& +\mathcal{M}\left\{\gamma \in \Gamma: M\left\|\eta_{k}(\gamma)-L_{2}(\gamma)\right\| \geq \beta-b\right\} .
\end{aligned}
$$

Since $\left\{\zeta_{k}\right\}$ and $\left\{\eta_{k}\right\}$ lacunary strongly convergent in measure to $L_{1}$ and $L_{2}$ respectively with respect to Orlicz function $M$.

So for $\varepsilon>0$ and $k \in I_{r}$ we have,

$$
\lim _{r \rightarrow \infty} \mathcal{M}\left\{\gamma \in \Gamma: \frac{1}{h_{r}} \sum_{k \in I_{r}} M\left\|\zeta_{k}(\gamma)-L_{1}(\gamma)\right\| \geq(\alpha-a) \geq \varepsilon\right\}=0
$$

and

$$
\lim _{r \rightarrow \infty} \mathcal{M}\left\{\gamma \in \Gamma: \frac{1}{h_{r}} \sum_{k \in I_{r}} M\left\|\eta_{k}(\gamma)-L_{2}(\gamma)\right\| \geq(\beta-b) \geq \varepsilon\right\}=0 .
$$

Thus we have, $\lim \sup _{r \rightarrow \infty} \Phi_{k}(c) \leq \Phi(\alpha+i \beta)$ for any $\alpha>a, \beta>b$.

Taking $\alpha+i \beta \rightarrow a+i b$, we get,

$$
\limsup _{r \rightarrow \infty} \Phi_{k}(c) \leq \Phi(c) .
$$

On the other hand, for any $x<a, y<b$ we have,

$$
\left\{L_{1} \leq x, L_{2} \leq y\right\}=\left\{\zeta_{k} \leq a, \eta_{k} \leq b, L_{1} \leq x, L_{2} \leq y\right\}
$$




$$
\begin{gathered}
\cup\left\{\zeta_{k} \leq a, \eta_{k} \leq b, L_{2} \leq x, L_{2} \leq y\right\} \\
\cup\left\{\zeta_{k}>a, \eta_{k} \leq b, L_{1} \leq x, L_{2} \leq y\right\} \cup\left\{\zeta_{k}>a, \eta_{k}>b, L_{1} \leq x, L_{2} \leq y\right\} \\
\subset\left\{\zeta_{k} \leq a, \eta_{k} \leq b\right\} \cup\left\{M\left\|\zeta_{k}-L_{1}\right\| \geq a-x\right\} \cup\left\{M\left\|\eta_{k}-L_{2}\right\| \geq b-y\right\} .
\end{gathered}
$$

which implies,

$$
\begin{aligned}
\Phi(x+i y) \leq & \Phi_{k}(a+i b)+\mathcal{M}\left\{\gamma \in \Gamma: M\left\|\zeta_{k}(\gamma)-L_{1}(\gamma)\right\| \geq a-x\right\} \\
& +\mathcal{M}\left\{\gamma \in \Gamma: M\left\|\eta_{k}(\gamma)-L_{2}(\gamma)\right\| \geq b-y\right\} .
\end{aligned}
$$

Since,

$$
\lim _{r \rightarrow \infty} \mathcal{M}\left\{\gamma \in \Gamma: \frac{1}{h_{r}} \sum_{k \in I_{r}}\left(M\left\|\zeta_{k}(\gamma)-L_{1}(\gamma)\right\| \geq a-x\right) \geq \varepsilon\right\}=0
$$

and

$$
\lim _{r \rightarrow \infty} \mathcal{M}\left\{\gamma \in \Gamma: \frac{1}{h_{r}} \sum_{k \in I_{r}}\left(M\left\|\eta_{k}(\gamma)-L_{2}(\gamma)\right\| \geq b-y\right) \geq \varepsilon\right\}=0,
$$

we obtain,

$$
\Phi(x+i y) \leq \lim \inf _{r \rightarrow \infty} \Phi_{k}(a+i b)
$$

for any $x<a, y<b$.

Taking $x+i y \rightarrow a+i b$, we get

$$
\Phi(c) \leq \liminf _{r \rightarrow \infty} \Phi_{k}(c) .
$$

It follows from (4.1) and (4.2) that $\Phi_{k}(c) \rightarrow \Phi(c)$ as $r \rightarrow \infty$ and $k \in I_{r}$. That is the complex uncertain sequence $\left\{\xi_{k}\right\}$ is lacunary strongly convergent in distribution to $L=L_{1}+i L_{2}$.

Converse of the above theorem is not necessarily true. i.e. Lacunary strongly convergent in distribution with respect to Orlicz function $M$ does not imply lacunary strongly convergence in measure with respect to that Orlicz function. Following example illustrates this.

Example 4.2. Consider the uncertainty space $(\Gamma, \mathcal{L}, \mathcal{M})$ to be $\left\{\gamma_{1}, \gamma_{2}\right\}$ with $\mathcal{M}\left\{\gamma_{1}\right\}=\mathcal{M}\left\{\gamma_{2}\right\}=\frac{1}{2}$. We define a complex uncertain variable as

$$
\xi(\gamma)= \begin{cases}1, & \text { if } \gamma=\gamma_{1} \\ -1, & \text { if } \gamma=\gamma_{2}\end{cases}
$$


We also define $\left\{\xi_{k}\right\}=-\xi$, for $k \in I_{r}$. Then $\left\{\xi_{k}\right\}$ and $\xi$ have the same distribution and thus $\left\{\xi_{k}\right\}$ converges in distribution to $\xi$. However, for any given $\varepsilon>0$, we have

$$
\begin{aligned}
& \lim _{r \rightarrow \infty} \mathcal{M}\left[\left\{\gamma \in \Gamma: \frac{1}{h_{r}} \sum_{k \in I_{r}} M\left\|\xi_{k}(\gamma)-\xi(\gamma)\right\|>\varepsilon\right\}\right] \\
& =\lim _{r \rightarrow \infty} \mathcal{M}\left[\left\{\gamma \in \Gamma: \frac{1}{h_{r}} \sum_{k \in I_{r}} M\left\|2 \xi_{k}(\gamma)\right\|>\varepsilon\right\}\right] \\
& \neq 0 .
\end{aligned}
$$

Therefore, the sequence $\left\{\xi_{k}\right\}$ does not lacunary strongly converge in measure to $\xi$ with respect to Orlicz function $M$.

Theorem 4.3. Let $\xi_{1}, \xi_{2}, \xi_{3}, \ldots$ be complex uncertain variables. Then $\left\{\xi_{k}\right\}$ is lacunary strongly convergent almost surely to $L$ with respect to an Orlicz function $M$ if and only if for any $\varepsilon>0$, we have,

$$
\mathcal{M}\left(\bigcap_{r \in I_{r_{k}}} \bigcup_{k \in I_{r}}\left\{\gamma \in \Gamma: \frac{1}{h_{r}} \sum_{k \in I_{r}} M\left\|\xi_{k}(\gamma)-L(\gamma)\right\|>\varepsilon\right\}\right)=0
$$

Proof. By the definition of lacunary strongly convergent almost surely we have that there exists an event $\Lambda$ with $\mathcal{M}(\Lambda)=1$, such that

$$
\lim _{r \rightarrow \infty} \frac{1}{h_{r}} \sum_{k \in I_{r}} M\left\|\xi_{k}(\gamma)-L(\gamma)\right\|=0
$$

for every $\gamma \in \Lambda$.

Then for any $\varepsilon>0$ there exists $m$ such that $\frac{1}{h_{r}} \sum_{k \in I_{r}} M\left\|\xi_{k}(\gamma)-L(\gamma)\right\|<\varepsilon$ where $k>m$, for any $\gamma \in \Lambda$, which is equivalent to

$$
\mathcal{M}\left(\bigcup_{r \in I_{r_{k}}} \bigcap_{k \in I_{r}}\left\{\gamma \in \Gamma: \frac{1}{h_{r}} \sum_{k \in I_{r}} M\left\|\xi_{k}(\gamma)-L(\gamma)\right\|>\varepsilon\right\}\right)=1 .
$$

But using the duality axiom it follows that

$$
\mathcal{M}\left(\bigcap_{r \in I_{r_{k}}} \bigcup_{k \in I_{r}}\left\{\gamma \in \Gamma: \frac{1}{h_{r}} \sum_{k \in I_{r}} M\left\|\xi_{k}(\gamma)-L(\gamma)\right\|>\varepsilon\right\}\right)=0 .
$$

Hence the result is proved.

Theorem 4.4. Let $\xi_{1}, \xi_{2}, \xi_{3}, \ldots$ be complex uncertain variables. If $\left\{\xi_{k}\right\}$ is lacunary strongly convergent uniformly almost surely to $L$ with respect to Orlicz function $M$, then $\left\{\xi_{k}\right\}$ is lacunary strongly convergent in measure to $L$ with respect to that Orlicz function $M$. 
Proof. If $\left\{\xi_{k}\right\}$ is lacunary strongly convergent uniformly almost surely to $L$ with respect to Orlicz function $M$ then

$$
\lim _{n \rightarrow \infty} \mathcal{M}\left(\bigcup_{k \in I_{r}}\left\{\gamma \in \Gamma: \frac{1}{h_{r}} \sum_{k \in I_{r}} M\left\|\xi_{k}(\gamma)-L(\gamma)\right\|>\varepsilon\right\}\right)=0
$$

from the above theorem.

But,

$$
\begin{gathered}
\mathcal{M}\left(\left\{\gamma \in \Gamma: \frac{1}{h_{r}} \sum_{k \in I_{r}} M\left\|\xi_{k}(\gamma)-L(\gamma)\right\|>\varepsilon\right\}\right) \\
\leq \mathcal{M}\left(\bigcup_{k \in I_{r}}\left\{\gamma \in \Gamma: \frac{1}{h_{r}} \sum_{k \in I_{r}} M\left\|\xi_{k}(\gamma)-L(\gamma)\right\|>\varepsilon\right\}\right) .
\end{gathered}
$$

Therefore, $\left\{\xi_{k}\right\}$ is lacunary strongly convergent in measure to $L$ with respect to Orlicz function $M$.

\section{Conclusion}

This paper introduces the different lacunary strongly convergent concepts of the sequences of uncertain variable with respect to Orlicz function. The results derived here generalizes the existing results.

\section{References}

[1] A. R. Freedman, J. J. Sember, and M. Raphael, "Some Cesàro-type summability spaces", Proceedings of the London Mathematical Society, vol. s3-37, no. 3, pp. 508-520, 1978. doi: 10.1112/ plms/ s3-37.3.508

[2] B. C. Tripathy and S. Mahanta, "On a class of sequences related to the $\ell^{p}$ space defined by Orlicz functions", Soochow journal of mathematics, vol. 29, no. 4, pp. 379-391, 2003.

[3] B. C. Tripathy and S. Mahanta, "On a class of generalized lacunary difference sequence spaces defined by Orlicz functions", Acta mathematicae applicatea sinica, english series, vol. 20, no. 2, pp. 231-238, 2004, doi: 10.1007/s10255-004-0163-1

[4] B. C. Tripathy and A. Esi, "Generalized lacunary difference sequence spaces defined by Orlicz functions", Matimyás matematika, vol. 28, no. 1-3, pp. 50-57, 2005. 
[5] B. C. Tripathy and P. J. Dowari, "Nörlund and Riesz mean of sequence of complex uncertain variables", Filomat, vol. 32, no. 8, pp. 2875-2881, 2018, doi: 10.2298/ FIL1808875T

[6] B. C. Tripathy and P. Nath, "Statistical convergence of complex uncertain sequences", New mathematics and natural computing, vol. 13, no. 2, pp. 359-374, 2017, doi: 10.1142/ S1793005717500090

[7] B. Liu, "Some research problems in uncertainty theory", Journal uncertain system, vol. 3, no. 1, pp. 3-10, 2009. [On line]. Available: https:/ / bit.ly/ 3pmVBqt

[8] B. Liu, "Why is there a need for uncertainty theory?", Journal uncertain system, vol. 6, no. 1, pp. 3-10, 2012. [On line]. Available: https:/ / bit.ly/ 3jV4xCl

[9] B. Liu, Uncertainty theory, 4th ed. Berlin: Springer, 2015, doi: 10.1007/ 978-3-662-44354-5

[10] C. You, "On the convergence of uncertain sequences", Mathematical and computer modelling, vol. 49, no. 3-4, pp. 482-487, 2009, doi: 10.1016/j.mcm.2008.07.007

[11] G. G. Lorentz, "A contribution to the theory of divergent sequences", Acta mathematica, vol. 80, pp. 167-190, 1948, doi: 10.1007/ BF02393648

[12] J. P. King, "Almost summable sequences", Proceeding American Mathematical Society, vol. 16, pp. 1219-1225, 1966, doi: 10.1090/ S0002-9939-1966-0201872-6

[13] P. J. Dowari and B.C. Tripathy, "Lacunary convergence of sequences of complex uncertain variables", Boletim da Sociedade Paranaense de Matemática (Online), Preprint, 2020, doi: 10.5269/ bspm.52688

[14] P. K. Kamthan and M. Gupta, Sequence spaces and series. New York, NY: M. Dekker, 1981.

[15] S. D. Parashar and B. Choudhary, "Sequence spaces defined by Orlicz functions", Indian journal of pure and applied mathematics, vol. 25, no. 4, pp. 419-428, 1994. [On line]. Available: https:/ / bit.ly/ 2ZD4QK

[16] X. Chen, Y. Ning, and X. Wang, "Convergence of complex uncertain sequences", Journal of intelligent \& fuzzy systems, vol. 30, no. 6, pp. 3357-3366, 2016, doi: 10.3233/ IFS-152083 